\title{
Toward community resources for paleoclimate data assimilation, reanalysis, and proxy system modeling
}

\author{
DAPS workshop participants* \\ Friends and members of the Data \\ Assimilation, Reanalysis and Proxy System \\ Modeling (DAPS; pastglobalchanges.org/ \\ daps) working group came together for \\ a brief meeting to discuss activities and \\ progress since our first meeting in Louvaine- \\ la-Neuve, Belgium, in May 2017.
}

\section{2nd DAPS workshop, College Park, MD, USA, 29-31 May 2019}

Data assimilation for paleoenvironmenta reconstruction: methods intercomparison Operational systems for weather to seasonal forecasting are now modular and open platforms, allowing for automated quality control, rejection of nonconforming observations, assessment of stochastic parameterizations, uniform multivariate skill assessment, and assessment of novel ap proaches such as use of future forecasts (see schematic Fig. 1). By comparison with the methods used in the DAPS Data Assimilation Intercomparison Project (DAIP), we identified similarities and differences between operational online data assimilation (DA) for weather and seasonal forecasting, and offline assimilation, optimal interpolation and linear regression/transfer function approaches to paleoclimatic reconstruction (Hakim et al. 2016; Franke et al. 2017). A key question, even for offline or timeindependent formulations, is whether and how to incorporate information at local versus remote scales in space. Can we trust the remote information, and at what level of filtering? Conversely, do we trust the local information? The answers likely depend on multiple approaches to skill estimation and validation of the results, at the process, data and parameter levels. Under some conditions, temporally aware data assimilation might improve results - processes for which timescales of variation are much longer than the timescales resolved by observations. For the present generation of paleo DA, this condition has not yet been met, but might be in the future, for analysis of variations associated with the intermediate and deep ocean, deep soil moisture, vegetation, and the cryosphere. These long timescales are at the heart of what might be gained from the exercise: identifying processes consistent with the observational evidence given uncertainties in all elements.

\section{Proxy system modeling: spatial} and structural considerations

We reviewed results from two Data Model Intercomparison projects (DMIP) across sensors, archives, and model complexity. Applying a bivariate linear model across marine carbonate archives identifies discrepancies between simulated and observed variance, which may be due to

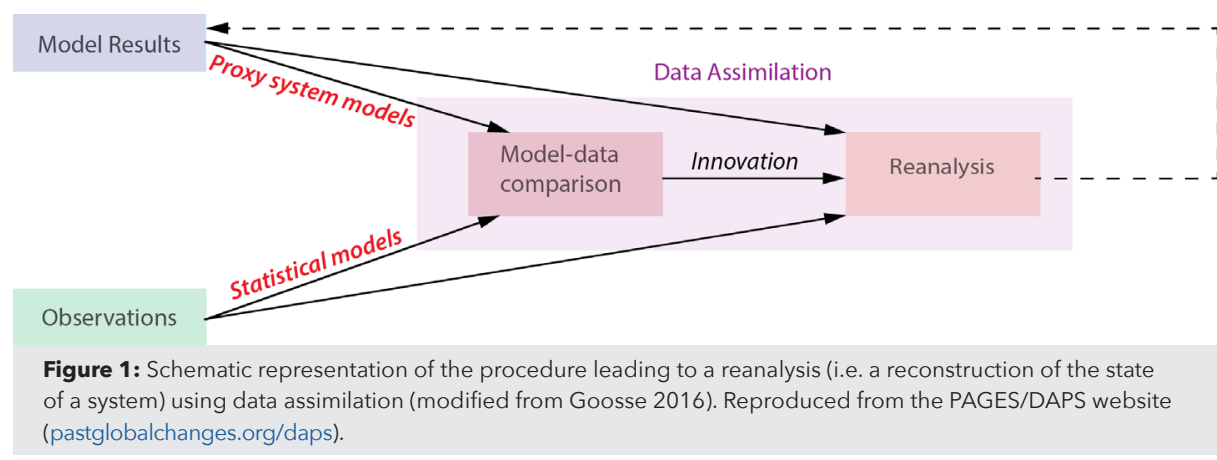

complex and variable growth responses. Limited validated information was retrieved from complex and nonlinear proxy system models producing tree-ring width simulations, and arose from modeling observations as bivariate indicators of both temperature and moisture variation, and under slowly changing mean climate states. However, conclusions might be sensitive to limitation by observational target and uncertainty, parameter estimation, timescale, and evalu ation metrics. For more complex processes and targets, more complex models might outperform simpler ones, but require additional inputs and parameters to be specified. At present, bivariate linear regression-based PSMs may be a good point of reference and null hypothesis on PSM complexity sufficient for use in paleo reanalysis products (Zhu et al. 2019a). Advances in unified platforms for proxy system modeling and evaluation, and for exploiting digitized paleodata and metadata via the Linked Paleo Data (LiPD; lipd. net) format (Dee et al. 2015; Zhu et al. 2019b) will enable more comprehensive studies and advances in the use of PSMs in paleoclimatic data assimilation exercises.

\section{Products and next steps}

An overarching theme that emerged from our discussions, fueled by nearly intravenous espresso and homemade food was that a common, open platform for development and assessment of approaches to paleoenvironmental data assimilation, reanalysis and proxy system modeling is sorely needed. As a result of a concentrated working session, such a community platform is being constructed (daps-pages.github. io) as a basis for ongoing work, and papers synthesizing DAIP, DMIP and challenges/ outlook are developing. However, DAPS was founded as a three-year project, and will sunset in 2019 - unless new leadership proposes a second phase! If you are interested in picking up on the themes and initiatives described here, please contact the group leaders (pastglobalchanges. org/science/wg/daps/people) for more information and suggestions for doing so.

\section{ACKNOWLEDGEMENTS}

M.N. Evans would like to thank the organizers of the PAGES2k-PMIP3 Workshop, Madrid, 2013, which produced such a vigorous and productive discussion of the questions arising from comparing paleodata and climate simulations; and which inspired our personalized DAPS nametags. Funding for ECR travel and accommodation was provided by PAGES; and for an outreach event (science.umd.edu/events/ scienceontap-2019-05.html), meeting space and refreshments by the University of Maryland, College Park, College of Mathematical and Natural Sciences, Earth System Science Interdisciplinary Center, Geology and Atmospheric and Oceanic Sciences Departments, and Reena Gupta.

\section{AFFILIATIONS}

${ }^{*}$ M.N. Evans (Univ. Maryland, College Park, USA); H. Goosse (Univ. Catholique de la Louvain, Belgium); J. Emile-Geay (USC, Los Angeles, USA); F. Zhu (USC Los Angeles, USA); S.G. Dee (Rice U., Houston, USA); J. Franke (Univ. Bern, Switzerland); J. Jeong (Vrie Univ. Amsterdam, Netherlands); J. Rezsöhazy (Univ. Catholique de la Louvain, Belgium); D.M. Thompson (Univ, Arizona, Tucson, USA); D. Amrhein (Univ. Washington, Seattle, USA); M.A. Cane (LDEO of Columbia University, Palisades, USA); A. Kaplan (LDEO of Columbia University, Palisades, USA): E. Kalnay (Univ. Maryland, College Park, USA); V. Tallapragada (NOAA/NCEP/DMAB, College Park, MD, USA); A. Okazaki (RIKEN, Kobe, Japan); A. Lawman (Univ. Texas, Austin USA); N. Wright (Australian National Univ., Canberra, Australia); S.H.S Herho (Bandung Institute of Technology, Indonesia): G. Schmidt (NASA/GISS, New York, USA); M.-F. Loutre (PAGES IPO, Bern, Switzerland); L. Parsons (Univ. Washington, Seattle, USA); J. Nadimikeri (Yogi Vemana Univ, Vemanapuram, India)

\section{CONTACT}

Michael N.Evans:mnevans@umd.edu

\section{REFERENCES}

Dee SG et al. (2015) J Adv Model Earth Sy 7: 1220-1247

Franke J et al. (2017) Sci Data 4: 170076

Goosse H (2016) J Adv Model Earth Sy 8: 1501-1503

Hakim GJ et al. (2016) J Geophys Res Atmos 121 6745-6764

Zhu F et al. (2019a) June 27 Zenodo

Zhu F et al. (2019b) May 22 Zenodo 